\title{
Article
}

\section{In-situ MIMO-WPT Recharging of UAVs using Intelligent Flying Energy Sources}

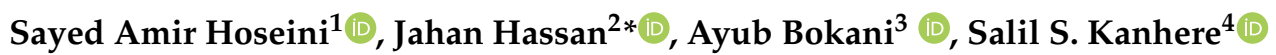 \\ 1 School of Engineering and Information Technology, The University of New South Wales, Canberra, \\ Australia ; s.a.hoseini@unsw.edu.au \\ 2,3 School of Engineering and Technology, The Central Queensland University, Sydney, Australia; \{j.hassan, \\ a.bokani\}@cqu.edu.au \\ 4 School of Computer Science and Engineering, The University of New South Wales, Sydney, Australia ; \\ s.kanhere@unsw.edu.au \\ * Correspondence: j.hassan@cqu.edu.au
}

\begin{abstract}
The Unmanned Aerial Vehicles (UAVs), used in civilian applications such as emergency medical deliveries, precision agriculture, wireless communication provisioning, etc., face the challenge of limited flight time due to their reliance on the on-board battery. Therefore, developing efficient mechanisms for in-situ power transfer to recharge UAV batteries hold potential in extending their mission time. In this paper, we study the use of far-field wireless power transfer (WPT) technique from specialized, transmitter UAVs (tUAVs) carrying Multiple Input Multiple Output (MIMO) antennas for transferring wireless power to receiver UAVs (rUAVs) in a mission. The tUAVs can fly and adjust their distance to the rUAVs to maximize energy transfer. The use of MIMO antennas further boost the energy reception by narrowing the energy beam toward the rUAVs. The complexity of their dynamic operating environment increases with the growing number of tUAVs, and rUAVs with varying levels of energy consumption and residual power. We propose an intelligent trajectory selection algorithm for the tUAVs based on a deep reinforcement learning model called Proximal Policy Optimization (PPO) to optimize the energy transfer gain. Simulation results demonstrate that with the use of PPO, the system achieves a tenfold flight time extension compared to no wireless recharging. Further, PPO outperforms the benchmark movement strategies of 'Traveling Salesman Problem' and 'Low Battery First' when used by the tUAVs.
\end{abstract}

Keywords: UAVs, Wireless Power Transfer, RF energy harvesting, MIMO, Deep Reinforcement Learning.

\section{Introduction}

Recent years have seen increasing advancements and decreasing costs of lowaltitude UAVs, commonly known as drones. Drones carrying a range of technologies for sensing and communication are becoming popular to service providers as innovative service delivery platforms, such as for emergency medical deliveries, precision agriculture, aerial imagery, etc. This popularity contributes directly to the growth of the global market for drone-delivered commercial services to an estimated value of $\$ 127 b n$ [1]. Drones are also employed in 5G networks either as aerial base stations providing wireless Hotspot or mobile relaying services to the ground nodes [2,3], or as aerial nodes of cellular UAV networks [4,5].

With such a staggering market value, reliability through service continuity becomes a critical success factor [6]. However, drones have short flying times due to their dependency on on-board, limited capacity batteries for power supply. For example, the typical flight-time of DJI Spreading Wings S900 drone is about 18 minutes when the battery is fully charged [3]. This implies that drones need to make frequent trips to 


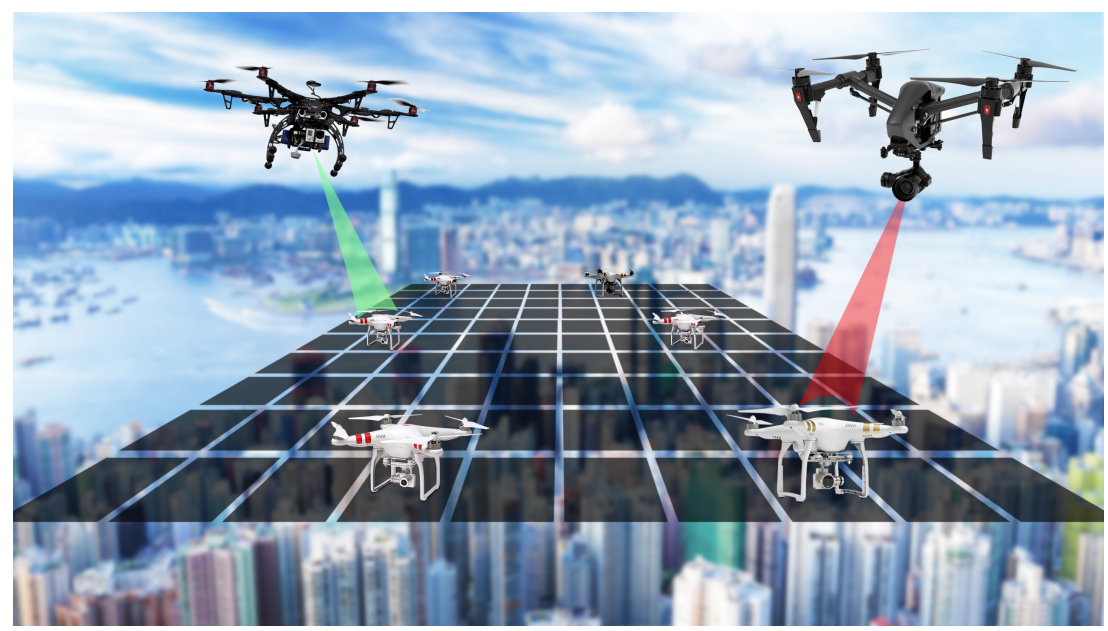

Figure 1. In-situ recharging of UAVs using aerial wireless energy sources: Sample Wireless Power Transfer (WPT) beams in a flying trajectory.

the ground charging stations to get their batteries replaced or recharged, which creates significant service disruptions. To reduce the disruption, in-situ recharging of the drone battery using ambient energy harvesting techniques is considered as a core technology for operational UAVs in the $5 \mathrm{G}$ networks [6].

The energy limitation issue is largely being addressed through the design and optimization of algorithms and motion control functions [7-12] to achieve energy efficiency. While such efforts are helpful, they do not fundamentally solve the problem since the drones would still require to fly away from their missions and return to ground charging stations when the battery eventually drains out. Solar powered drones can harvest energy from the sun. To harvest enough energy, the drones need fixed wings with a long wingspan (e.g., 4 meters [13]) to accommodate the solar panels. As such, smaller, consumer drones as well as rotary-wing drones cannot benefit from this solution. Moreover, the solar energy harvesting is dependent on flight conditions, e.g., cloudy days or night times are not favorable for this type of energy harvesting. The far-field WPT using Radio Frequency (RF) Electromagnetic Radiation (EMR) technique (radiative WPT) is a promising approach for powering UAVs [14,15], which allows the transmitter and receiver being located over a distance. This specifically suits the deployment and mobility requirements of recharging UAVs during missions.

The viability of the far-field WPT approach was demonstrated in [16-19]. William C. Brown for instance, showed how a wireless-powered helicopter can be powered over a distance of $18 \mathrm{~m}$ above the transmitting antenna in 1964 [16]. In this experiment, $270 \mathrm{~W}$ power could be harvested at $2.45 \mathrm{GHz}$. Recently, the simultaneous wireless information and power transfer system was explored to send wireless information and power to drones from terrestrial base stations $[20,21]$. However, the drones need to remain in the close proximity of the base stations during the WPT process to achieve Line-of-Sight (LoS) links. This limits the deployment location and mobility of the UAVs. Other issues with far-field WPT is the drop in transmission efficiency due to high path loss when the distance between the transmitter and receiver is increased [15], and the random, uncontrollable energy arrival at the receiver when non-dedicated energy sources are used [6].

In this paper, therefore, we propose the deployment of multiple, flying energy transmitters for recharging UAVs (rUAVS) using WPT. This is inspired by the practice of mid-air fueling of military jets using aerial tankers, a concept that was also proposed for civil aviation purpose [22]. The transmitters are specialized UAVs (tUAVs) equipped with Multiple-Input-Multiple-Output (MIMO) antennas. The MIMO antenna system can direct energy beams towards the receivers. The rUAVs can continue their mission without having to adjust their locations to receive power, while the tUAVs dynamically 
adjust their locations to reduce the transmission distance for enhancing the power delivery. To boost the WPT efficiency while meeting the regulatory constraints on maximum transmission power [23], in each $\mathrm{tUAV}$, we propose to deploy multiple antennas that operate in multiple bands spectrum. This is because the use of multi-band transmission helps to distribute power over a wider spectrum so that the maximum transmitted power does not exceed the regulated limit. The rUAVs convert the received RF power to DC power using an array of rectennas that are special type of antennas used to convert electromagnetic energy to DC current. A conceptual view of our proposal is shown in Figure 1.

The energy consumption of each rUAV may be different due to the environmental conditions (e.g., windy or still conditions), dynamic wireless communication requirements, and mobility. Therefore, the tUAVs must intelligently pick the rUAVs to serve according to their residual energy levels, in a coordinated manner. This calls for a multi-agent optimization model, for which we employ Proximal Policy Optimization (PPO), a recent class of Deep Reinforcement Learning (DRL) algorithms. PPO adjusts each $\mathrm{tUAV}$ 's movements to intelligently pick the next rUAVs to be recharged considering traveling time, other rUAVs locations, rUAVs battery level and the other tUAVs location. This minimizes the service interruptions by extending flying times of the rUAVs. In other words, using PPO, each $\mathrm{tUAV}$ can find the best location to move to at a given observation of entire network of rUAVs. To the best of our knowledge, this is the first attempt to consider such a wireless charging architecture for UAVs using multiple dedicated, and coordinating aerial energy sources.

The main contributions of this paper can be summarized as: (i) we propose a system of multiple $\mathrm{tUAV}$ s to facilitate aerial wireless charging of rUAVs using multi-band MIMO beamforming, (ii) we propose a PPO-based movement decision algorithm for the tUAVs in selecting the next rUAVs to recharge as per their battery levels, and (iii) we compare the PPO-based system performance using simulations, with two benchmark movement decisions strategies of the tUAVS traveling Salesman Problem (TSP) and Low Battery First $(L B F)$. Our results demonstrate that with PPO, the system achieved a tenfold flight time extension compared to no WPT. Further, this strategy outperforms the benchmark movement strategies of TSP and LBF when used with WPT.

The rest of the paper is organized as follows. The related works are discussed in Section 2. System description and the DRL model is presented in Section 3 followed by the performance evaluation of proposed model in Section 4 . We finally conclude the paper and discuss future works in Section 5.

\section{Related Works}

\subsection{Energy-Efficient UAVs}

Researchers commonly address the drone energy-limitation issue through the design of their energy-efficient functioning mechanisms. These mechanisms include flight path (trajectory) planning and communication methods. UAVs consume energy due to their mechanical (flying, hovering) and electronic (wireless communication) functions, presenting scopes for improving energy-efficiency of both.

As the mechanical energy consumption is significantly more than that from the electronic functions, researchers mostly focus on optimizing the trajectory to shorten the flight paths for reducing mechanical energy consumption, e.g., in [7]. However, since saving energy, no matter how little, is important for battery-constrained UAVs, in a prior work we presented an optimization of the UAV trajectory to minimize the movement related energy consumption, while also reducing the communication energy consumption through the adaptive selection of a right radio frequency band for the specific application instead of using the same band for all applications [8]. Other researchers proposed a method to reduce the overall energy consumption of UAV communications by extending their network lifetime while guaranteeing their communication's success rate [9]. Optimal data collection techniques [10,11] and a UAV-aided networking mechanism [12] 
were also proposed that positively affect the UAV's energy consumption by optimizing their networking and communication methods.

\subsection{Far-field Wireless Powering of UAVs}

As already mentioned, there have been many trials in the past demonstrating the viability of far-field WPT techniques. Further development and practical use of such techniques somewhat stalled, however, we see a renewed interest as evident in recent industry needs, activities and trials. A New-Zealand based startup company, Emrod, was recently reported to have developed a long-range, high-power, WPT technology to deliver wireless electricity to end users without needing copper power lines [24]. This follows by the country's second largest power company Powerco, planing to trial the technology in 2021 [25]. In 2020, a US-based company, Powerlight Technologies (formerly known as LaserMotive), demonstrated a wireless power receiver for drones. in an earlier demonstration, the company used a laser beam to fly a drone for more than 12 hours [26].

The above mentioned recent demonstrations aside, the demand for solutions to autonomous wireless recharging of drones is further evident in the numerous commercial missions that the drones are increasingly being used recently, e.g., in powerline monitoring (e.g., [27]), food delivery (e.g., [28]) and law enforcement (e.g., [29]), to name a few. The short flight times of these drones are causing serious deployment hindrances in these industries. According to a 2020 Bloomberg report, the predicted market value of the global autonomous wireless charging and infrastructure market for drones will reach $\$ 249.3$ Million by 2024 [30]. This justifies the unified efforts from research and industry sector that is required to develop practical solutions for wireless charging of drones, more importantly, in-situ solutions.

The utility of far-field WPT using EM radiation is well established: it provides placement flexibility and mobility of transmitters and receivers, can work even in nonLoS conditions, and can power over a distance. Due to the energy conversion efficiency limitation, generally this technique is suitable for low-power devices. Despite this limitation, various works on WPT suggest that far-field WPT can also be used for recharging UAV batteries (e.g., [15]). To this end, wireless recharging of UAVs were proposed using RF WPT in [20] and optical energy transfer in [21], both from ground base stations in a simultaneous wireless information and power transfer system. Using a power-splitting and time-switching architecture, authors proposed a relaying system in [20] in which the UAV harvests energy and information from the base station, and relay the information to a ground node. With an objective of prolonging the lifetime and throughput maximization of the network, authors optimized the system parameters along with the UAV deployment location, however no explicit results on received power was mentioned. Authors in [21] studied a similar system but with an optical transmitter at the ground base station casting optical beam to the UAV carrying both data and energy, providing simultaneous communication and charging. Numerical results showed that the system achieved a high network throughput and a $25 \%$ extra hovering time in the drones. However, both proposals require the UAVs to be in the proximity of the terrestrial base station to achieve LoS and receive power. This limits the locations where the UAVs can be deployed due to the fixed terrestrial base stations. Therefore, flexible in-situ wireless charging of UAVs remains a challenging open problem.

In our previous works [31,32], we studied different modes of dedicated, aerial WPT chargers to observe their performance subsequently, with tUAVs carrying omnidirectional antennas. In [31] we utilized aerial, stationary (i.e., hovering at fixed locations) tUAVs to study their optimal placement locations with respect to the rUAVs to maximize total received power at the rUAVs. In [32], we utilized one flying tUAV to power all rUAVs. In that work, for the single tUAV to recharge all rUAVs, a single-agent optimization of the tUAV's trajectory was presented via Q-Learning to enhance power delivery. However, Q-Learning comes with a scalability issue and both observation and action space must 
be limited. As such, for multi-agent systems (current work) Q-learning poses limitations. Further, the use of omnidirectional antennas waste energy since energy is radiated all around the antenna, not only to the energy receiver. The fundamental differences of our current work with our prior works is that our current work uses multiband MIMO antennas at the flying energy sources which distributes power over a wider spectrum and exploits targeted energy beams through beamforming to recharge chosen UAVs. This boosts energy delivery at the rUAVs. Further, we employ a multi-agent optimization model for multiple tUAVs in the network to optimize the tUAVs' movement decisions using the PPO algorithm. The advantages of PPO over Q-Learning is discussed in the next section.

\section{System Description}

In this section, we present our UAV recharging architecture involving multiple tUAVs and rUAVs. We also present the Deep Reinforcement Learning algorithm using the PPO technique to control the movements of the tUAVs in targeting the next rUAVs to recharge. The optimization aims to enhance the MIMO-WPT efficiency to achieve longer flying times of the rUAVs in the presence of multiple coordinated tUAVs serving multiple rUAVs with dynamic battery levels.

\subsection{UAVs Recharging Architecture}

Our proposed UAVs recharging architecture consists of specialized, flying UAVs equipped with multiple high gain RF antennas (tUAV) that transmit wireless power to recharge the rUAVs' batteries. We assume that the rUAVs are deployed in an area to provide Hotspot wireless communication services to the ground users (Figure 1). The tUAVs are assumed to have sufficient power supply, e.g., by carrying a larger battery, or having hybrid power sources. The tUAVs fly about and position themselves in a way to minimize the distance between them and the rUAVs, and to improve the line-of-sight RF links for the target rUAVs. This enhances the power transfer effectiveness.

To increase energy transfer efficiency, we propose a MIMO system to perform an energy beamforming and focus energy toward the receiver [33-35]. Hence, we consider a point-to-point MIMO system with $m_{t}$ antennas installed on the tUAVs and $m_{r}$ antennas on the rUAVs. Without the loss of generality, we assume a uniform square array of antenna on each side which is also mechanically alignable. We use the system model obtained from $[14,34]$ where a total of $N$ orthogonal sub-bands are used to transmit energy. On each sub-band, sine-wave signal $\mathbf{S}_{n}$ is emitted at carrier frequency $f_{n}$ by $m_{t}$ tUAV antennas as

$$
\mathbf{s}_{n}(t)=\left[s_{1 n}(t), \ldots, s_{m_{t} n}(t)\right]^{T},
$$

where $n=1,2, \ldots, N$ and $s_{m n}(t)$ is the beamforming component of $s_{n}(t)$ by antenna $m$ at frequency $n$. Thus the total received power at all $m_{r}$ receiver antennas is defined as

$$
P_{\mathrm{r}}=\sum_{i=1}^{m_{r}} \sum_{n=1}^{N} \mathbb{E}\left[\left|\mathbf{h}_{\mathbf{i n}}^{\mathbf{H}} \mathbf{s}_{n}(t)\right|^{2}\right]=\sum_{n=1}^{N} \operatorname{tr}\left(\mathbf{H}_{n}^{H} \mathbf{H}_{n} \mathbf{S}_{n}\right),
$$

where $\mathbf{h}_{i n}^{H}\left[h_{i 1 n^{\prime}}^{*}, \cdots, h_{i m_{t} n}^{*}\right]$ is the channel vector from transmitter antennas to receiver antenna $i, \mathbf{H}_{n}$ is the channel matrix between $m_{t}$ transmitter antennas and $m_{r}$ receiver antennas and $\mathbf{S}_{n}$ is the transmit covariance matrix all at sub-band $n$. Similarly, the total transmit power at frequency $f_{n}$ is

$$
P_{\mathrm{t}}=\sum_{n=1}^{N} \operatorname{tr}\left(\mathbf{S}_{n}\right)
$$


The maximum transmit power at each sub-band is constrained by regulation and hardware limits. Thus, let us assume

$$
\operatorname{tr}\left(\mathbf{S}_{n}\right) \leq P_{s}, \forall n
$$

Based on [14] and assuming the maximum sum-power $P_{s}$ is transmitted at each sub-band, the received power at each sub-band $n$ is obtained as

$$
P_{\mathrm{r}, \mathrm{n}}=P_{\mathrm{s}} \lambda_{\max , n}^{2}, n=1, \cdots, N,
$$

where $\lambda_{\max , n}=\lambda_{\max }\left(\mathbf{H}_{n}^{H} \mathbf{H}_{n}\right)$ denotes the maximum singular value of $\mathbf{H}_{n}^{H} \mathbf{H}_{n}$ for subband $n$. As a result, the total harvested energy is

$$
P_{\mathrm{r}}=P_{S} \sum_{n=1}^{N} \lambda_{\max , n}^{2}
$$

Since there is almost a pure LoS MIMO channel between a pair of $\mathrm{tUAV}$ and rUAV during the recharging process as the tUAV adjusts its position to achieve this, $\mathbf{H}_{n}$ is a rank one matrix and an optimal beamforming can be achieved by an SVD-based beamformer [36,37] when only one strong beam is formed by transmitter antennas as optimal energy beamforming with a gain of $n_{t}[34,38]$,

$$
\lambda_{\max , n}=a_{n} \sqrt{m_{t} m_{r}},
$$

where $a_{n}$ is the signal attenuation along the LoS path at frequency $n$ which is assumed to be the same for all antenna pairs. This assumption is valid when the distance between transmitter and receiver is much larger than the antenna array size[36]. For this purpose, the Channel State Information (CSI) should be available at transmitter side. In contrast with MIMO channel's information, the energy transfer channel in our system is significant, stable and relatively time invariant. Hence, measuring the CSI feedback is not a challenging task. Attenuation is also obtained by

$$
a_{n}^{2}=\frac{G_{t} G_{r} c^{2}}{\left(4 \pi d f_{n}\right)^{2}}
$$

where $G_{t}$ and $G_{r}$ are each antenna gain at transmitter and receiver respectively, $c$ is the speed of light and $d$ is the distance between the transmitter and receiver. Thanks to mechanical alignment, high gain antennas can be employed to boost MIMO gain [38,39]. We applied a limit of $90 \%$ efficiency [40] to the RF gain in (5) to model nonideal implementation of MIMO system, i.e. mutual coupling. There is also RF to DC conversion efficiency at receiver which represents how much of received wireless energy can be converted to usable energy by the rUAVs. In this work, we assume a constant RF to DC efficiency of $80 \%[41,42]$ that is denoted by $\gamma$.

\subsection{Proposed Trajectory Selection Algorithm}

Proximal policy optimization (PPO) is a model-free, online, on-policy reinforcement learning method from policy gradient family $[43,44]$. This method supports both discrete and continuous spaces for observations and actions. A PPO agent transitions from one state to another, by taking random actions. A set of states $S$ which are defined based on the observations from the environment and a set of actions $A$ define the learning space. By performing an action $a \in A$ and observing the resulting state, a revenue function calculates a numeric reward. The learner's goal is to maximize the discounted long-term reward state-action pairs from beginning up to reaching the goal state, so called the optimal policy. The optimal policy indicates which action is the best to take in different states, which results in a maximized overall gain. PPO selects actions based on the probability distribution and we define the optimal policy so that the action with 
maximum likelihood is chosen after training as deterministic exploitation. PPO finds the best location and movement for the tUAVs at a given observation of entire network of rUAVs.

Reinforcement learning has been widely used in UAV related research recently. This includes a range of application from military threat avoidance [45] and obstacle avoidance [46] to trajectory optimization for improving services in wireless communications [47]. In our previous work [32], we used Q-Learning which is not scalable for large observation space and multi-agent systems. Hence, we employ PPO with discrete observation and action space. PPO also allows us to use fine-grained discrete observation values where this can be an implementation issue in Q-Learning that is limited by the Q-table size. PPO components in our solution are defined as the following:

- Agent (tUAV) observes the current state and takes actions. There can be multiple agents in our scenario. To keep model simple, we implement multiple tUAV system as a single agent PPO with multiple actions.

- $\quad$ State $(S)$ is defined based on the observed information of rUAVs and the current location of tUAV. Thus, we define the system state as $S=\left\{L_{c}, L_{h}, B_{h}\right\}$ where $L_{c}$ is the location of tUAVs, $L_{h}=\left[L_{h_{1}}, L_{h_{2}}, \ldots, L_{h_{Z}}\right]$ is a vector that denotes the locations of rUAV1 to rUAVz and $B_{h}=\left[B_{h_{1}}, B_{h_{2}}, \ldots, B_{h_{Z}}\right]$ is a vector that denotes their battery levels.

- Action $(a)$ is defined as flying to hovering above certain rUAVs. Hence, number of possible actions is equal to number of rUAVs. PPO algorithm implements a function approximator $\mu(S)$ that takes state $S$ and returns the probabilities of taking each action in the action space.

- $\quad$ Revenue $(R)$ is the combination of rewards and penalties after taking action $a$ at state $S$ and moving to state $S^{\prime}$. It returns a reward for the energy that all rUAVs receive from $\mathrm{tUAV}$ and/or applies a penalty if an rUAV has to move to terrestrial charging station because of low battery. $R$ is formulated as:

$$
R\left(S, a, S^{\prime}\right)=w_{1} \int_{T} P_{r} \mathrm{~d} t+w_{2} N_{o}+w_{3} B_{l}+w_{4} B_{f}+w_{5} Q
$$

where $w_{1}, \ldots, w_{5}$ are adjusting weights, $P_{r}$ is the total harvested power by rUAVs noted in (5), $T$ is the time step and $N_{o}$ is the number of out of charge rUAVs which should be replaced and resulted in service interruption. $B_{l}$ represent the low battery thresholds of rUAVs and is defined as

$$
\begin{aligned}
B_{\text {low }} & =\sum_{k=1}^{Z} \bar{B}_{k}, \quad \text { while } \\
\bar{B}_{k} & = \begin{cases}0.05 B_{\max }-B_{h_{k}} & \text { if } B_{h_{k}} \leq 0.05 B_{\max }, \\
0 & \text { otherwise }\end{cases}
\end{aligned}
$$

where $B_{\max }$ is the battery capacity of rUAV. $B_{f}$ denotes the full battery if the battery is more than $97 \%$ charged. Finally $Q$ indicates the conflict between the tUAVs if their distance from each other is less than a threshold. This can force them to not charge the same rUAV in the same time and also avoid a crash. The second PPO function approximator is the critic $V(S)$ that takes observation $S$ and returns the expectation of the discounted long-term reward [43,44].

In the above model, each agent (tUAV) needs to observe all rUAVs' geographical locations and their remaining battery levels. We assume that the rUAVs remain in the same geo-cell in our considered area, therefore, only their battery status needs to be sent to the tUAVs at each time step. Hence, our tUAVs and rUAVs must have a light periodic signalling to exchange information. 

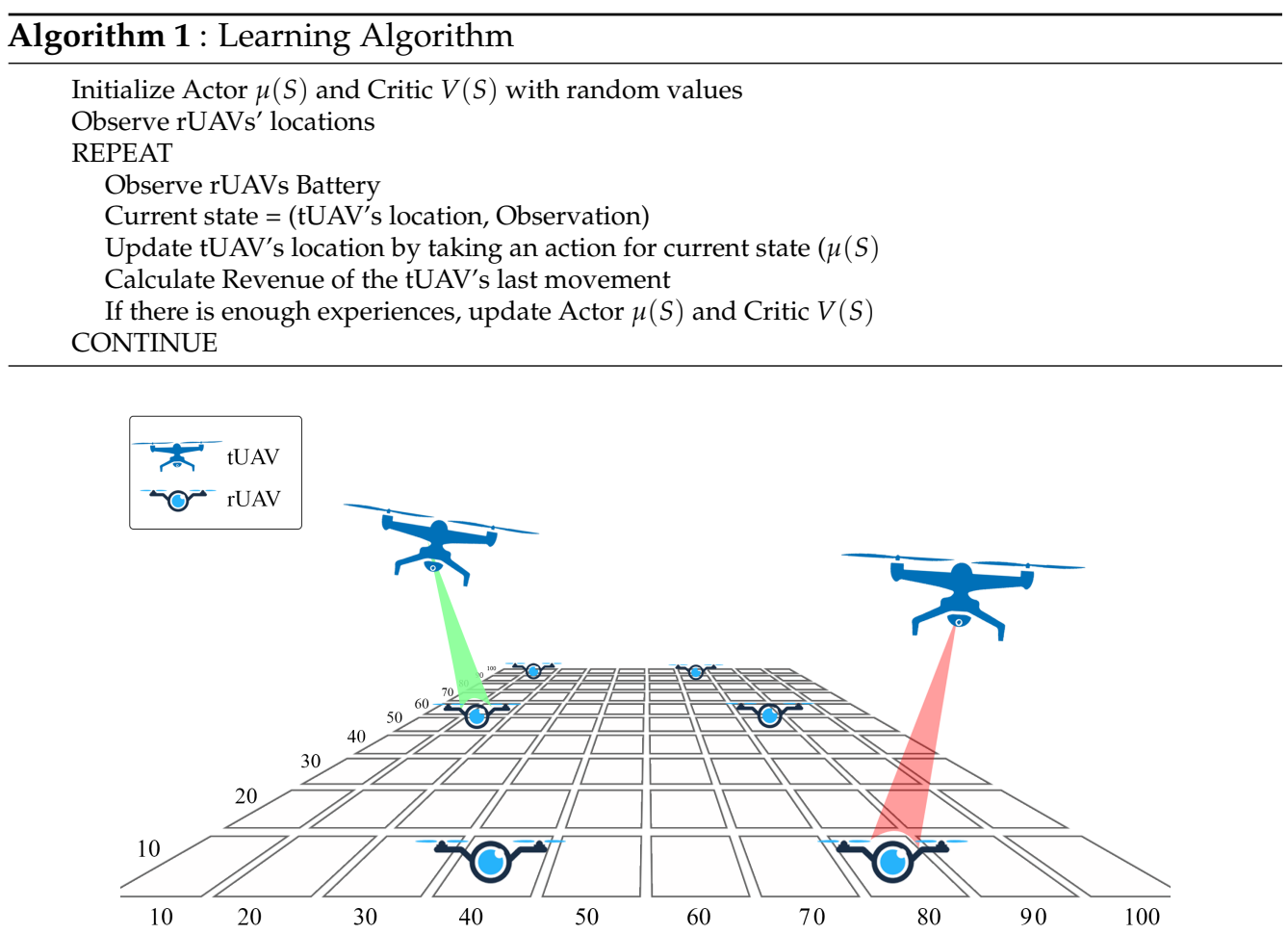

Figure 2. Considered simulation scenarios showing tUAVs and rUAVs positions. The rUAVs are stationary in each scenario and tUAVs periodically change position to improve the energy transfer efficiency where the initial position of $\mathrm{tUAV}$ is randomized for each episode.

Considering the discussed Reinforcement Learning components, we follow Algorithm 1 to obtain an optimal flying trajectory (i.e., movement decisions) of the tUAVs and recharging mechanism that maximizes the overall flying duration of all rUAVs. In this algorithm, each $\mathrm{tUAV}$ receives updated information of the rUAVs at each time step. The observation includes the tUAVs current location indicating the current state. The agent makes a decision on movement based on the actor output. Recharging is considered only when the $t U A V$ arrives to hover above the chosen rUAV because recharging is assumed inefficient when tUAV is flying. The PPO details are not presented in 1 as it can be found in $[43,44]$. The algorithm can be executed centrally in a ground control station or by the tUAVs individually.

\section{Performance Evaluation}

In this section, we first describe the simulation set-up including the baseline algorithms that we compare the PPO's performance against. We then present and discuss key results of this research. 


\subsection{Simulation Setup}

Table 1. Simulation Parameters

\begin{tabular}{|l|l|}
\hline Simulation Component & Value \\
\hline Transmit power of each sub-band $P_{s}$ & 1 Watt \\
Antenna element gain $G_{t}, G_{r}$ & $16 \mathrm{dBi}$ \\
Number of antenna on $\mathrm{tUAV} m_{t}$ & 256 \\
Number of antenna on rUAV $m_{r}$ & 256 \\
Number of sub-bands $N$ & 200 \\
Sub-band's width & $10 \mathrm{MHz}$ \\
Cell side & $10 \mathrm{~m}$ \\
Charging Wave Frequency range & $25-27 \mathrm{GHz}$ \\
Learning rate & 0.4 \\
Discount factor & 0.95 \\
rUAV power consumption & $50 \pm 10 \mathrm{Watt}$ \\
rUAV battery capacity & 30 Watt - hour $(108 \mathrm{~kJ})$ \\
Time step & 30 or more Sec \\
Revenue adjusting weights $\left(w_{1}, w_{2}, w_{3}, w_{4}, w_{5}\right)$ & $0.001,-10000,-0.0001,-0.00003,-10000$ \\
\hline
\end{tabular}

In our scenarios, we consider six rUAVs and two tUAVs, located in an environment modeled as a $100 \times 100$ grid (Figure 2). To simplify our simulation design, we assume all rUAVs can be located only at the center of cells as illustrated in Figure 2. Each $\mathrm{tUAV}$ sends the recharging beam toward the target rUAV that is selected by the algorithm. We selected arbitrary frequency of $25-27 \mathrm{GHz}$ which can be adjusted as per the spectrum regulations in the region. Note that increasing the frequency increases free space path loss but more antennae can be installed in the same antenna size since MIMO proper inter-element space is related to wave length. We assume a maximum power of 1 Watt is transmitted at each sub-band of $10 \mathrm{MHz}$ width. This is reasonable in terms of regulations as in most of countries mobile devices that work in millimeter-wave spectrum are permitted to operate in $83 \mathrm{dBm} / 100 \mathrm{MHz}$ range [23]. There are 256 antenna elements installed on each $\mathrm{tUAV}$ and $\mathrm{rUAV}$ in a uniform square array, and since the EM wavelength is about $1.2 \mathrm{~cm}$, the array can be readily fitted on a small drone. For a square array number of antenna elements should be a power of 2, e.g., 256.

In order to evaluate our algorithm's performance, we use MATLAB Reinforcement Learning Toolbox to simulate the environment and implement the PPO algorithm. Additionally, we simulate the following two benchmark schemes for the tUAVs' movement decisions:

- Traveling Salesman Problem (TSP): Each tUAV recharges a group of 3 rUAVs periodically and in order. The groups and orders should be selected so that the traveling times of the tUAVs are minimized. We solve the TSP using an iterative approach to find the best two groups to be served by the two tUAVs.

- $\quad$ Lowest Battery First (LBF): The tUAVs target to serve the rUAVs with the minimum battery level at each time step.

To compare the performance of the PPO and the above baseline schemes and also to show the WPT recharging effect, we count the number of times that an rUAV battery reaches to the minimum threshold and it is replaced with a full battery rUAV after few seconds. The rUAV replacement can result in a service interruption to the nodes that are served by the respective rUAV (e.g., in the Hotspot service scenario). As such, the replacements should be minimized. Also, we calculate the average flying time of all rUAVs. We assumed that the WPT recharging in our scenario is not enough to keep all rUAVs in service for long time. This is because the total recharging power is less than consumed power. A period of 10 hours is simulated to study the impact of the recharging. 


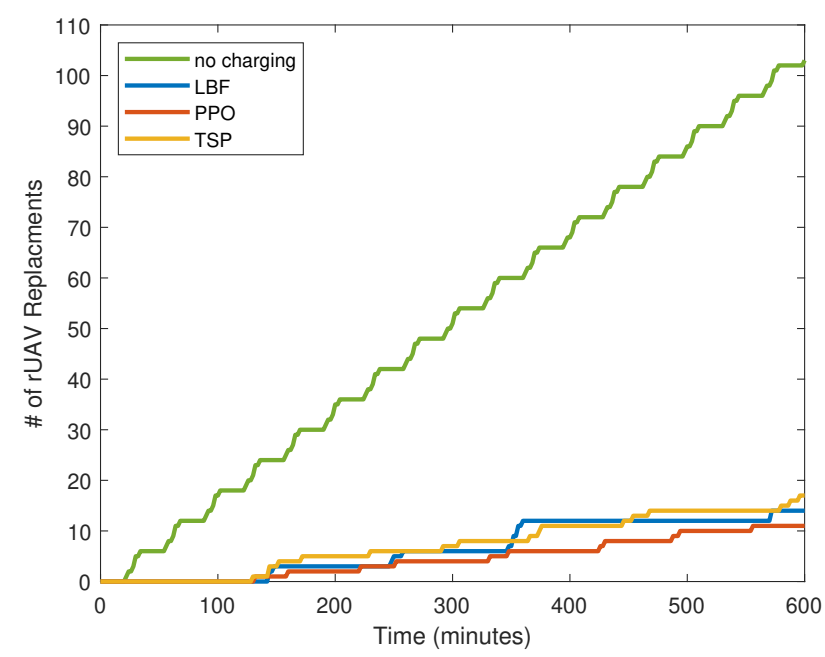

Figure 3. Comparison of number of rUAV replacements when an rUAV is out of charge.

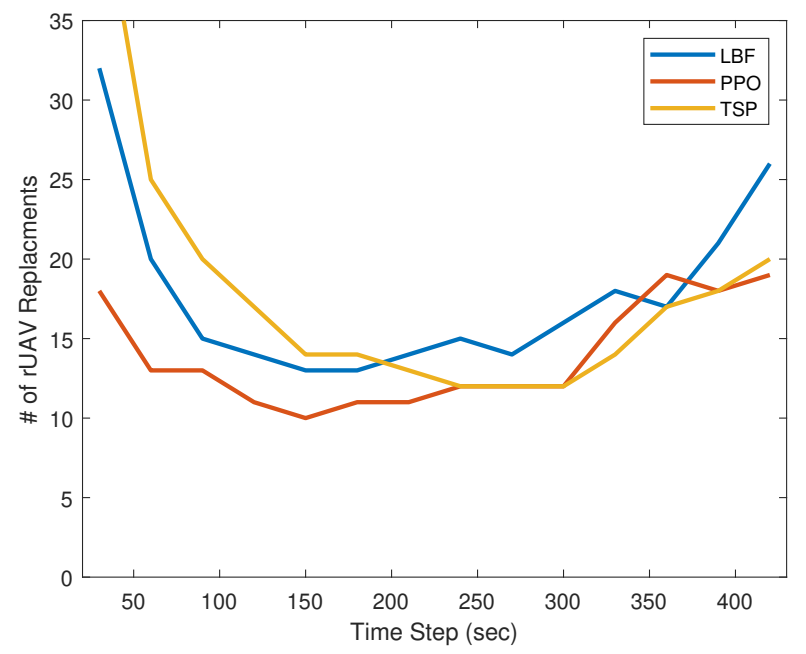

Figure 4. Comparison of different tUAV movement schemes used with the WPT for different time step values. Each tUAV takes the next action to update the location at the end of each time step.

\subsection{Results}

In this section, we present the simulation results based on the above system model and algorithm.

First, we run simulations with and without WPT capability to see the viability of proposed model. We can evaluate both systems' performance based on the number of times rUAVs need to be replaced due to the battery depletion. As it's illustrated in Figure 3, using MIMO based WPT-enabled tUAVs, significantly improves the system performance by reducing the number of rUAV replacements from 108 (when no WPT recharging is used) to less than 20. In the same simulation, we also evaluated the performance of proposed algorithm against benchmark moving models, all with WPT enabled tUAVs. As it can be seen from Figure 3, PPO outperforms the benchmark schemes where in 10 hours duration, the number of rUAV replacements is only 11 in comparison with 14 and 17 for LBF and TSP schemes. We assumed 120 second time steps for tUAVs to retake decisions in this simulation.

Second, since the tUAV's recharging ability is not used when it is traveling, increasing the time step may improve the results for benchmarks. For this purpose, we simulated the scenario with different time step duration to compare different models' 


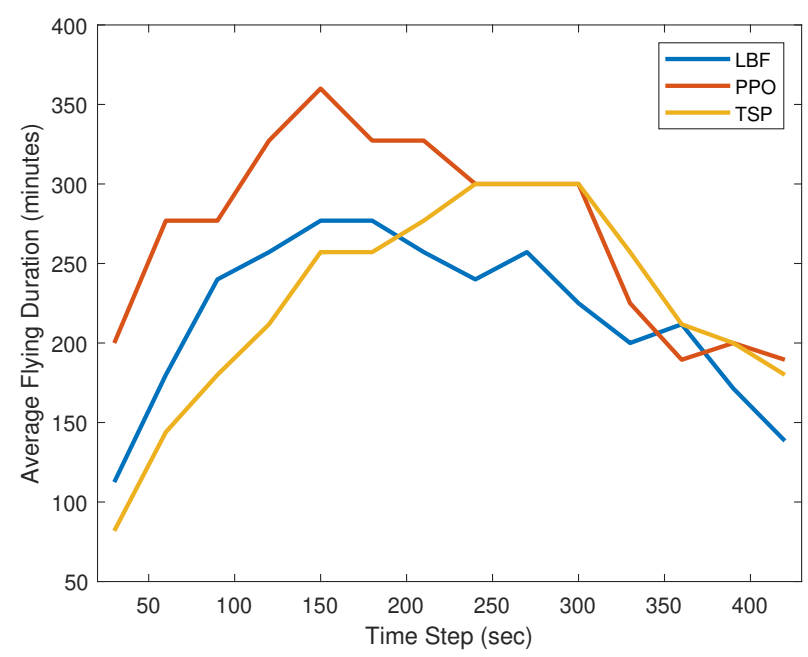

Figure 5. Average flying time of rUAVs for different time step values. In the absence of WPT recharging, the average flying time is 33 minutes.

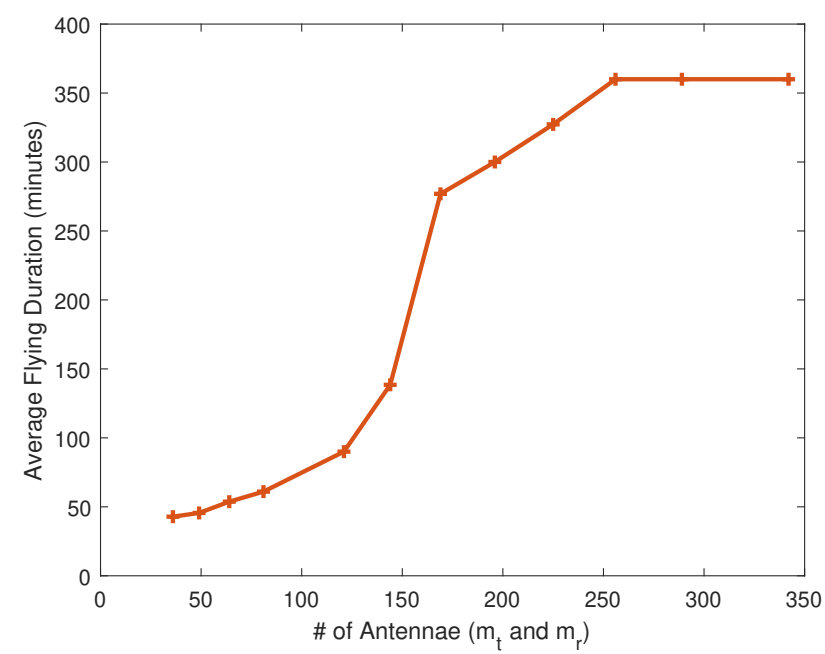

Figure 6. Average flying time of rUAVs for different antennae numbers when PPO is used.

performance. The result is plotted in Figure 4. As it can be seen, the PPO's performance also can be improved for longer time steps of about 150-200 seconds. It is further observed that TSP can be as good as PPO for some time steps. However, the figure shows that the PPO performance superiority is kept for all time step values despite that the gap is reduced in 150-200 seconds. The best performance is recorded for 150 seconds when only 10 replacements are recorded over 10 hours. The best performance of TSP is recorded as 12 when time step is 240-300 seconds.

We have recorded the flight duration of all rUAVs in our simulation. Figure 5 demonstrates their average flying times. As it's shown, while it is only 33 minutes without employing WPT recharging mechanism, the proposed PPO based WPT enabled system can increase rUAVs' flight duration up to 360 minutes for the studied scenario. Moreever, it can be observed that the low complexity schemes of LBF and TSP can achieve 280 and 300 minutes flying duration that is notable. This result demonstrates the merit of our proposed MIMO-WPT based UAVs recharging architecture, irrespective of the specific movement strategy of the tUAVs. However, PPO's performance achieves notable gains of about $20-25 \%$ higher than the benchmark movement schemes used by the tUAVs. 
On the other hand, we present the average flight duration of rUAVs in Figure 6 for different number of antennas in which we consider the same value for $m_{r}$ and $m_{t}$. Clearly, increasing the number of antenna elements can increase the beamforming gain and improve the WPT efficiency. However, this is limited by the total transmitting power, and the WPT's efficiency (RF-RF) cannot be more than $100 \%$. Note that we assumed a maximum of $90 \%$ RF-RF efficiency to address the non-ideal implementation factors such as mutual impedance between antenna elements in the antenna array [40].

\section{Conclusion and Future Work}

We studied the concept of using dedicated, flying chargers equipped with MIMO antenna for in-situ recharging of UAVs' batteries using wireless power transfer. We formulated the movement decision of the aerial chargers to recharge the UAV s as a multiagent optimization problem using the Proximal Policy Optimization (PPO) to optimize the energy transfer gain and enhance the UAVs' flying times. Using simulation studies, we demonstrated that the PPO algorithm provided a tenfold flight time extension for the deployed system compared to no wireless recharging of the UAVs. Further, PPO outperformed the benchmark schemes, when used with our proposed MIMO WPT system. Although we simulated with a scenario of Hotspot UAVs that hover above fixed locations, it can be generalized for all applications where the power receiver UAVs are hovering above a certain location, e.g., for traffic monitoring at an intersection. Future work could consider other applications with mobile UAVs as power receivers, and the use of hybrid power sources at the chargers.

\section{Acknowledgment}

This work was supported by the Central Queensland University Research Grant RSH5137.

\section{References}

1. Silver, B.; Mazur, M.; Wisniewski, A.; Babicz, A. Welcome to the era of drone-powered solutions: a valuable source of new revenue streams for telecoms operators. https://www.pwc.com/gx/en/communications/pdf/communications-review-july-2017.pdf, 2017. Accessed: 2020-03-10.

2. Fotouhi, A.; Ding, M.; Hassan, M. Flying Drone Base Stations for Macro Hotspots. IEEE Access 2018, PP, 1-1. doi:10.1109/ACCESS.2018.2817799.

3. Fotouhi, A.; Qiang, H.; Ding, M.; Hassan, M.; Giordano, L.G.; Garcia-Rodriguez, A.; Yuan, J. Survey on UAV Cellular Communications: Practical Aspects, Standardization Advancements, Regulation, and Security Challenges. IEEE Communications Surveys Tutorials 2019, 21. doi:10.1109/COMST.2019.2906228.

4. Zhang, R.; Zheng, Y. Accessing from the Sky: UAV Communications for 5G and Beyonds (Tutorial). IEEE International Conference on Communications (ICC) (Tutorial). IEEE, 2019.

5. Zeng, Y.; Lyu, J.; Zhang, R. Cellular-Connected UAV: Potential, Challenges, and Promising Technologies. IEEE Wireless Communications 2019, 26, 120-127.

6. Li, B.; Fei, Z.; Zhang, Y. UAV Communications for $5 \mathrm{G}$ and Beyond: Recent Advances and Future Trends. IEEE Internet of Things Journal 2019, 6, 2241-2263. doi:10.1109/JIOT.2018.2887086.

7. Tran, D.H.; Vu, T.; Chatzinotas, S.; Shahbazpanahi, S.; Ottersten, B. Coarse Trajectory Design for Energy Minimization in UAV-Enabled. IEEE Transactions on Vehicular Technology 2020, 69, 9483-9496.

8. Salehi, S.; Bokani, A.; Hassan, J.; Kanhere, S.S. AETD: An Application Aware, Energy Efficient Trajectory Design for Flying Base Stations. 2019 IEEE 14th Malaysia International Conference on Communication (MICC), 2019.

9. Li, K.; Ni, W.; Wang, X.; Liu, R.P.; Kanhere, S.S.; Jha, S. Energy-efficient cooperative relaying for unmanned aerial vehicles. IEEE Transactions on Mobile Computing 2015, 15, 1377-1386.

10. Abdulla, A.E.; Fadlullah, Z.M.; Nishiyama, H.; Kato, N.; Ono, F.; Miura, R. An optimal data collection technique for improved utility in UAS-aided networks. IEEE INFOCOM 2014-IEEE Conference on Computer Communications. IEEE, 2014, pp. 736-744.

11. Zhan, C.; Zeng, Y.; Zhang, R. Energy-efficient data collection in UAV enabled wireless sensor network. IEEE Wireless Communications Letters 2017, 7, 328-331.

12. Abdulla, A.E.; Fadlullah, Z.M.; Nishiyama, H.; Kato, N.; Ono, F.; Miura, R. Toward fair maximization of energy efficiency in multiple UAS-aided networks: A game-theoretic methodology. IEEE Transactions on Wireless Communications 2014, 14, 305-316.

13. Morton, S.; D'Sa, R.; Papanikolopoulos, N. Solar powered UAV: Design and experiments. 2015 IEEE/RSJ International Conference on Intelligent Robots and Systems (IROS), 2015, pp. 2460-2466. doi:10.1109/IROS.2015.7353711. 
14. Zeng, Y.; Clerckx, B.; Zhang, R. Communications and Signals Design for Wireless Power Transmission. IEEE Transactions on Communications 2017, 65, 2264-2290. doi:10.1109/TCOMM.2017.2676103.

15. Huang, J.; Zhou, Y.; Ning, Z.; Gharavi, H. Wireless Power Transfer and Energy Harvesting: Current Status and Future Prospects. IEEE Wireless Communications 2019, 26, 163-169. doi:10.1109/MWC.2019.1800378.

16. Brown, W.C. Experiments involving a microwave beam to power and position a helicopter. IEEE Transactions on Aerospace and Electronic Systems 1969, pp. 692-702.

17. Shinohara, N. Beam control technologies with a high-efficiency phased array for microwave power transmission in Japan. Proceedings of the IEEE 2013, 101, 1448-1463.

18. Strassner, B.; Chang, K. Microwave power transmission: Historical milestones and system components. Proceedings of the IEEE 2013, 101, 1379-1396.

19. Jull, G.W.; Lillemark, A.; Turner, R. SHARP (stationary high altitude relay platform) telecommunications missions and systems. GLOBECOM'85-Global Telecommunications Conference, 1985, Vol. 2, pp. 955-959.

20. Hua, M.; Li, C.; Huang, Y.; Yang, L. Throughput Maximization for UAV-enabled Wireless Power Transfer in Relaying System. 2017 9th International Conference on Wireless Communications and Signal Processing (WCSP), 2017, pp. 1-5. doi:10.1109/WCSP.2017.8170970.

21. Ansari, N.; Wu, D.; Sun, X. FSO as backhaul and energizer for drone-assisted mobile access networks. ICT Express 2020.

22. Nangia, R.K. 'Greener' civil aviation using air-to-air refuelling - relating aircraft design efficiency and tanker offload efficiency. The Aeronautical Journal (1968) 2007, 111, 589-592. doi:10.1017/S0001924000001858.

23. Commission, F.C. FCC-Use of Spectrum Bands Above 24 GHz For Mobile Radio Services. https://apps.fcc.gov/edocs_public/ attachmatch/FCC-16-89A1.pdf, 2016. Accessed: 2021-07-07.

24. Shukla, H. This Wireless Power Technology Could Change New Zealand's Transmission System. https://mercomindia.com/ this-wireless-new-zealand-transmission-system/, 2020. Accessed: 2021-06-23.

25. Delbert, C. The Dawn of Wireless Electricity Is Finally Upon Us. Here's How New Zealand Will Do It. https://www. popularmechanics.com/science/a33522699/wireless-electricity-new-zealand/, 2021. Accessed: 2021-06-23.

26. Boyle, A. PowerLight is hitting its targets with a power beaming system that uses lasers. https://www.geekwire.com/2021 /powerlight-hitting-targets-power-beaming-system-uses-laser-light/, 2021. Accessed: 2021-06-23.

27. Bennett, T. TransGrid deploys drones to perform power line work. https://www.itnews.com.au/news/transgrid-deploysdrones-to-perform-power-line-work-559095, 2020. Accessed: 2021-06-23.

28. Wing. https://wing.com/en_au/australia/canberra/. Accessed: 2021-06-23.

29. Metz, C. Police Drones Are Starting to Think for Themselves. https://www.nytimes.com/2020/12/05/technology/policedrones.html, 2020. Accessed: 2021-06-23.

30. Banga, B. Global Autonomous Drone Wireless Charging and Infrastructure Market to Reach \$249.3 Million by 2024. https: / / www.bloomberg.com/press-releases/2020-01-07/global-autonomous-drone-wireless-charging-and-infrastructuremarket-to-reach-249-3-million-by-2024, 2020. Accessed: 2021-06-23.

31. Hassan, J.; Bokani, A.; Kanhere, S.S. Recharging of Flying Base Stations using Airborne RF Energy Sources. 2019 IEEE Wireless Communications and Networking Conference Workshop (WCNCW), 2019, pp. 1-6. doi:10.1109/WCNCW.2019.8902900.

32. Hoseini, S.A.; Hassan, J.; Bokani, A.; Kanhere, S.S. Trajectory Optimization of Flying Energy Sources using Q-Learning to Recharge Hotspot UAVs. IEEE INFOCOM 2020 - IEEE Conference on Computer Communications Workshops (INFOCOM WKSHPS), 2020, pp. 683-688. doi:10.1109/INFOCOMWKSHPS50562.2020.9162834.

33. Xu, J.; Bi, S.; Zhang, R. Multiuser MIMO Wireless Energy Transfer With Coexisting Opportunistic Communication. IEEE Wireless Communications Letters 2015, 4, 273-276. doi:10.1109/LWC.2015.2407357.

34. Xu, J.; Zhang, R. A General Design Framework for MIMO Wireless Energy Transfer With Limited Feedback. IEEE Transactions on Signal Processing 2016, 64, 2475-2488. doi:10.1109/TSP.2016.2526965.

35. Wang, Y.; Liu, A.; Xu, K.; Xia, X. Energy and Information Beamforming in Airborne Massive MIMO System for Wireless Powered Communications. Sensors 2018, 18, 3540 .

36. Tse, D.; Viswanath, P. Chapter 07: MIMO I : spatial multiplexing and channel modeling. Fundamentals of Wireless Communication 2005, pp. 290-331.

37. Hoseini, S.A.; Ding, M.; Hassan, M.; Chen, Y. Analyzing the Impact of Molecular Re-Radiation on the MIMO Capacity in High-Frequency Bands. IEEE Transactions on Vehicular Technology 2020, 69, 15458-15471. doi:10.1109/TVT.2020.3041488.

38. Hamdy, M.N. Beamformers Explained. https://www.commscope.com/globalassets/digizuite/542044-Beamformer-ExplainedWP-114491-EN.pdf, 2020. Accessed: 2021-07-07.

39. Agrawal, T.; Srivastava, S. Two element MIMO antenna using Substrate Integrated Waveguide (SIW) horn. 2016 International Conference on Signal Processing and Communication (ICSC), 2016, pp. 508-511. doi:10.1109/ICSPCom.2016.7980633.

40. Aoki, T.; Yuan, Q.; Quang-Thang, D.; Okada, M.; Hsu, H.M. Maximum transfer efficiency of MIMO-WPT system. 2018 IEEE Wireless Power Transfer Conference (WPTC). IEEE, 2018, pp. 1-3.

41. Carvalho, A.; Carvalho, N.; Pinho, P.; Goncalves, R. Wireless power transmission and its applications for powering Drone. Article for the 8th Congress of the Portuguese Committee of URSI, 2014.

42. Brown, W.C. The history of wireless power transmission. Solar Energy 1996, 56, 3-21. Wireless Power Transmission, doi:https:/ / doi.org/10.1016/0038-092X(95)00080-B. 
43. Schulman, J.; Wolski, F.; Dhariwal, P.; Radford, A.; Klimov, O. Proximal policy optimization algorithms. arXiv preprint arXiv:1707.06347 2017.

44. MathWorks. Proximal Policy Optimization Agents. https://www.mathworks.com/help/reinforcement-learning/ug/ppoagents.html. Accessed: 2021-05-30.

45. Yan, C.; Xiang, X.; Wang, C. Towards Real-Time Path Planning through Deep Reinforcement Learning for a UAV in Dynamic Environments. Journal of Intelligent \& Robotic Systems 2019. doi:10.1007/s10846-019-01073-3.

46. Yijing, Z.; Zheng, Z.; Xiaoyi, Z.; Yang, L. Q learning algorithm based UAV path learning and obstacle avoidence approach. 2017 36th Chinese Control Conference (CCC). IEEE, 2017, pp. 3397-3402.

47. Challita, U.; Saad, W.; Bettstetter, C. Deep reinforcement learning for interference-aware path planning of cellular-connected UAVs. 2018 IEEE International Conference on Communications (ICC). IEEE, 2018, pp. 1-7. 